\title{
Aging workforce of United States physicians - something to ponder
}

\author{
Muhammad Nabeel* \\ Department of Medicine, Michigan State University, USA
}

\begin{abstract}
The inflamed dilemma of physician burns out, constantly evolving healthcare industry, growing stressors of reimbursement, health information technology and surfeit of compliance requirements all are placing the millennials and post-millennial physicians under a tremendous strain. It is probable that such an exposure to high intensity stressors lead to premature exhaustion of physician workforce manifesting in sub-standard care delivery and poor health outcomes at the population level. Having a pro-active approach to look at some of the challenges faced by the graying workforce will benefit the entire physician cohort of the future. In this article, I take the stance that we need to engage in a comprehensive dialogue towards policy making of how to best manage the challenges of graying physician, how population health will be affected, how we can preserve a valuable experienced physician capitol and best utilize it to meet the demands of patients without compromising safety. Senior physicians bring valuable clinical expertise and life experiences acquired through years of practice however at the same time, we must acknowledge the limitations accrued with natural aging process. It is imperative that we keep our focus on high quality care and not just on quantity of care delivered.
\end{abstract}

\section{Introduction}

About one in four U.S. physicians are older than 65, and the number of doctors in this age bracket more than quadrupled between 1975 and 2013 [1]. Actively licensed physicians who are 60 years of age or older represent the single largest age category of physicians (31\%, up from $26 \%$ in 2012) [2]. Although age, in itself, does not imply incompetence, the cognitive function does begin to decline from age 55 and cognitive decline is quite prevalent among older physicians who are referred for competency evaluations $[3,4]$. In current healthcare environment, there is growing emphasis on "physician burn out", "patient safety", "value-based care", "health information technology" and "integration of care", therefore at the same time we must remain cognizant of the disproportional workforce of aging physicians asked to carry out these challenging tasks for the populace. Patient safety advocates, consumer groups, and policy makers have questioned whether older physicians maintain the necessary cognitive and motor skills to provide safe and competent care [4]. A systematic review published in 2005 found an inverse relationship between years of practice and several measures of quality, suggesting that older physicians might be at risk of providing lower-quality care [5]. It is not surprising that certain complex procedures (pancreatectomy, coronary artery bypass grafting procedures, and carotid endarterectomy) when performed in declining volume and by aging physicians have higher morbidity and mortality [6]. While physicians get evaluated during residency and fellowship training in multiple competencies, but once they become credentialed and licensed to practice independently, ongoing licensure status is maintained through continuing medical education (CME) credits and credentialing through an absence of significant issue identified by respective institution accreditation program requirements. In 2007, the Joint Commission introduced its Ongoing Professional Practice Evaluation (OPPE) and Focused Professional Practice Evaluation (FPPE) processes. These tools were created to work together to help determine if the care delivered by a provider falls below an acceptable level of performance. OPPE is a screening tool to evaluate all practitioners who have been granted privileges and to identify clinicians who might be delivering an unacceptable quality of care. FPPE is the follow-up process to determine the validity of any positives (whether true or false) found through OPPE, FPPE is applied only to the small number of clinicians identified through OPPE [7]. The value of OPPE depends on the measures selected by the medical staff; these measures represent activities related to quality of care and performance. Organizations which have collected meaningful data in a continuum and provide that data to individual practitioners (particularly if accompanied by peer or benchmark data) have found that providers become actively engaged both in validating the information and using it to adjust their practice. The effort of collecting reliable data, measuring various outcomes, interpreting the data and analyzing the information has become more common over last decade however there remains significant heterogeneity between independent small community practices and large health care organizations. With a growing trend towards consolidation in healthcare marketplace, there is a good likelihood that information collection, analysis and learning processes will improve.

\section{Why address the concern and what can be done?}

The ultimate goal of a competency assessment program is to improve patient safety, reduce cost, and enhance the population health [3]. It is unclear if OPPE and FPPE are well equipped to handle the challenges that health industry faces with increasingly aging physician population. Interesting analogy with some parallels can be considered with the profession of airline pilot where the Fair Treatment for Experienced Pilots Act mandates that pilots 60 years and older must

Correspondence to: Muhammad Nabeel, Department of Medicine, Michigan State University, East Lansing, USA, Tel: 517-353-3730, E-mail: muhammad. nabeel@hc.msu.edu

Received: April 01, 2018; Accepted: April 16, 2018; Published: April 20, 2018 
obtain a "first-class medical certificate" and mandatory renewal every six months. The medical screening requires testing of hearing, eyesight and an electrocardiogram (EKG). Arguably, this program allows for ongoing testing of a pilot's cognitive and physical capabilities [8]. In a small practice setting like many independent community practices there are significant limitations in implementing OPPE and FPPE. Even for larger healthcare systems though the quality measures can be obtained, it's not unexpected to find feasibility challenges in monitoring provider performance on a continuous basis. As health care systems move towards breaking silos and enhancing team approaches in delivering care it is all together more important to establish transparent evaluation triggers one of which can be conditioned on physician's advanced age. Physicians must develop guidelines and standards for monitoring and assessing both their own and their colleagues' competency. Formal guidelines on the timing and content of testing for competence can be deliberated and, by being more transparent with a standard evaluation process the aging physicians can continue to practice without a fear of worsening limited supply of physician workforce.

\section{Impact on Generation Y (millennial) and Generation Z (post-millennial) physicians}

Aging workforce has become a serious problem in the disproportionally aging cohort of the U.S. healthcare providers. A national survey demonstrated that $96 \%$ of physicians agreed that impaired or incompetent colleagues should be reported. However, only $45 \%$ physicians reported their colleagues when encountered with an alarming situation [9]. American Medical Association Council on Medical Education has been following this area of concern however there is lack of a consistent policy, just relying on self-regulation and institutional policies is insufficient. Aging workforce challenge is compounded by the major concern of physician burnout which affects nearly half of practicing physicians in the United States at some point in their career [10]. In the current healthcare workforce, a significant proportion of physicians belong to the millennial/postmillennial generation of both education and training however the recent slew of changes in the practice of medicine, growing concern of burn out, constant changes in reimbursement models and plethora of work related stressors are predisposing even the young physicians to premature graying effects often times manifesting as cutting down on FTE, exacerbating provider dissatisfaction, frequent changing of employers, early retirement etc. There is a significant opportunity for younger physicians to engage and learn from the challenges faced by senior physicians and at the same time the age of 65 must be considered as a trigger point into a "Physician Professional Competency Assessment Program." The program should include an annual peerevaluation, history and physical with cognitive evaluation, assessment for health information technology competence, and participation in a developed simulation model for procedure-driven specialties (as these become available and validated from specialty-specific boards). For physicians identified with potential deficits, in-depth evaluation by specialized centers should be done within a reasonable timeframe. If deficiencies are identified a mechanism should be put in place to limit privileges until the identified condition is satisfactorily resolved. Some of the strategies that can be adopted include less call/overnight shifts, reduction in clinical FTE status, job sharing, junior faculty mentoring, assigning teaching responsibilities and increased downtime between shifts [11]. There are limitations to these alternative options however as the industry landscape transitions towards integration, consolidation and contractual alliances these limitations are likely to diminish in the long run. All alternatives must be considered in context of keeping patient safety an utmost priority and must not be viewed as an effort to exacerbate physician supply chain. Inarguably we need to find a balance; a center point where we do not go overboard at the same time we must not ignore the fundamental job description of a physician as described in the Hippocratic oath "first do no harm". Developing and administering a policy addressing this concern requires multi-level approach, the good news is that various physician organizations like American Medical Association (AMA) have already generated some consensus on the issue. In the Report 5 put out by AMA in 2015, Council of Medical Education concluded that physicians should be allowed to remain in practice if patient safety is not endangered and that, if needed, remediation should be a supportive, ongoing and proactive process [12].

\section{Conclusion}

All individuals age, however the rate of change in executive function, wisdom, memory, and other components of cognition are not linear, they often fluctuate over time, and can be influenced by a variety of factors independent of age. The dearth of research studies looking at the association between aging physicians and quality of care along with anecdotal evidence of cognitive decline in older physicians behooves policymakers, researchers and healthcare leaders to prioritize and invest resources in mitigating this challenge [3,4]. There may not be a perfectly desirable solution to the problem; however, taking logical steps in safeguarding patient's optimum care, collecting data, performing meaningful analysis followed by development of robust strategy will be an excellent start. Senior physicians bring valuable skills, clinical expertise, and life experiences which is acquired by years of practice. However, at the same time, we must acknowledge the limitations accrued with the natural aging process. Safety and quality of care delivered to patients is of paramount importance, further research is needed to look in depth of this very important issue. It is imperative that we keep our focus on high quality care and not just the quantity of care the U.S. healthcare system delivers.

\section{References}

1. Competency and retirement: Evaluating the senior physician [Internet]. [Available from: http://www.ama-assn.org/ama/ama-wire/post/competency-retirement-evaluating-senior-physician]

2. Young A, Chaudhry H, Pei X, Arnhart K, Dugan, M, et al. (2014) A census of actively licensed physicians in the United States. J Med Regul 103: 11-24.

3. Kupfer JM (2015) The graying of us physicians: Implications for quality and the future supply of physicians. JAMA 315: 341-342. [Crossref]

4. Korinek LL, Thompson LL, McRae C, Korinek E (2009) Do physicians referred for competency evaluations have underlying cognitive problems? Acad Med 84: 10151021. [Crossref]

5. Powell DH (1994) Profiles in Cognitive Aging. 251.

6. Choudhry NK, Fletcher RH, Soumerai SB (2005) Systematic Review: The Relationship between Clinical Experience and Quality of Health Care. Ann Intern Med 142: 260273. [Crossref]

7. Waljee JF, Greenfield LJ, Dimick JB, Birkmeyer JD (2006) Surgeon age and operative mortality in the United States. Ann Surg 244: 353-362. [Crossref]

8. (2013) OPPE and FPPE: Tools to help make privileging decisions $\mid$ Joint Commission [Internet]. Joint Commission. [Available from: https://www.jointcommission.org jc_physician_blog/oppe_fppe_tools_privileging_decisions/]

9. Shanafelt TD, Hasan O, Dyrbye LN, Sinsky C, Satele D, et al. (2015) Changes in Burnout and Satisfaction with Work-Life Balance in Physicians and the Genera US Working Population Between 2011 and 2014. Mayo Clin Proc 90: 1600-1613. [Crossref]

10. Fair Treatment of Experienced Pilots Act (The Age 65 Law) [Internet]. [Available from $\mathrm{http}: / /$ avstop.com/legal/fair_treatment_of_experienced_pilots_act.htm] 
11. Takakuwa KM, Biros MH, Ruddy RM, FitzGerald M, Shofer FS (2013) A national survey of academic emergency medicine leaders on the physician workforce and institutional workforce and aging policies. Acad Med 88: 269-275. [Crossref]
12. (2015) AMA Report 5 Council of Medical Education (A-15) - Competency and Aging Physician.

Copyright: (C2018 Nabeel M. This is an open-access article distributed under the terms of the Creative Commons Attribution License, which permits unrestricted use, distribution, and reproduction in any medium, provided the original author and source are credited. 